\title{
Ginsenoside Rg1 ameliorates hippocampal long-term potentiation and memory in an Alzheimer's disease model
}

\author{
FENGLING LI ${ }^{1}$, XIQING WU ${ }^{2},{\text { JING } \mathrm{LI}^{3} \text { and QINGLIANG NIU }}^{2}$ \\ ${ }^{1}$ Department of Neurology, The Affiliated Hospital of Weifang Medical University, Weifang, Shandong 261031; \\ ${ }^{2}$ Medical Imaging Center; ${ }^{3}$ Department of Orthopaedics Rehabilitation, Weifang Traditional Chinese Medicine Hospital, \\ Weifang, Shandong 261041, P.R. China
}

Received April 24, 2015; Accepted March 2, 2016

DOI: $10.3892 / \mathrm{mmr} .2016 .5103$

\begin{abstract}
The complex etiopathogenesis of Alzheimer's disease (AD) has limited progression in the identification of effective therapeutic agents. Amyloid precursor protein (APP) and presenilin-1 (PS1) are always overexpressed in $\mathrm{AD}$, and are considered to be the initiators of the formation of $\beta$-amyloid plaques and the symptoms of AD. In the present study, a transgenic AD model, constructed via the overexpression of APP and PS1, was used to verify the protective effects of ginsenoside $\operatorname{Rg} 1$ on memory performance and synaptic plasticity. AD mice (6-month-old) were treated via intraperitoneal injection of $0.1-10 \mathrm{mg} / \mathrm{kg}$ ginsenoside $\mathrm{Rg} 1$. Long-term memory, synaptic plasticity, and the levels of AD-associated and synaptic plasticity-associated proteins were measured following treatment. Memory was measured using a fear conditioning task and protein expression levels were investigated using western blotting. All the data was analyzed by one-way analysis of variance or t-test. Following 30 days of consecutive treatment, memory in the AD mouse model was ameliorated in the $10 \mathrm{mg} / \mathrm{kg}$ ginsenoside Rg1 treatment group. As demonstrated by biochemical experiments, ginsenoside $\mathrm{Rg} 1$ treatment reduced the accumulations of $\beta$-amyloid 1-42 and phosphorylated (p)-Tau in the AD model. Additionally, brain-derived neurotrophic factor (BDNF) and p-TrkB synaptic plasticity-associated proteins were upregulated following ginsenoside Rg1 application. Correspondingly, long-term potentiation (LTP) was restored following ginsenoside $\mathrm{Rg} 1$ application in the AD mice model. Taken together, ginsenoside Rg1 repaired hippocampal LTP and memory, likely through facilitating the clearance of $\mathrm{AD}$-associated proteins and through activation of the BDNF-TrkB pathway.
\end{abstract}

Correspondence to: Dr Qingliang Niu, Medical Imaging Center, Weifang Traditional Chinese Medicine Hospital, 1055 Weizhou Road, Weifang, Shandong 261041, P.R. China

E-mail: qinglian_niu@163.com

Key words: Alzheimer's disease, ginsenoside Rg1, long-term potentiation, brain-derived neurotrophic factor/tropomyosin receptor kinase B
Therefore, ginsenoside Rg1 may be a candidate drug for the treatment of $\mathrm{AD}$.

\section{Introduction}

Alzheimer's disease (AD) remains the most severe form of neurodegenerative disease, and is characterized by a decline in memory performance and other cognitive abilities. It is estimated that $\sim 25,000,000$ individuals are affected worldwide, particularly in the elderly population (1). At present, there is no cure for $\mathrm{AD}$, however, certain symptomatic therapeutics are available (2-4). Although the etiology of AD remains to be fully elucidated, there is a general consensus in favor of the plaque hypothesis. The hallmark appearance of amyloid plaques and intracellular neurofibrillary tangles of Tau are reported to contribute to neuron loss in $\operatorname{AD}(5,6)$. The formation of $\beta$-amyloid plaques in $\mathrm{AD}$ patients affects neuronal synaptic plasticity in the early phase, and progressively leads to cell death in the later phase (7-9). Therefore, plaque formation and synaptic plasticity, particularly in the hippocampus, which is a region responsible for memory formation, are critical indices in evaluating anti-AD efficacy.

The use of Chinese medicines has a long-term history in clinical practice, and has been suggested to offer potential in improving memory $(10,11)$. Among the effective herbs, ginseng is considered to promote the health of middle-aged and elderly populations (12). Ginseng has been used as an adaptogenic herb in traditional Chinese medicine for $>2,000$ years, and the long-term application of ginseng improves the ability to combat stress, trauma, anxiety and fatigue (13). Additional pharmacological activities, including in the prevention of cancer and neurodegenerative diseases, have also been reported $(14,15)$. Ginsenosides, the active compounds of the Panax species, have been widely investigated in basic and clinical settings. A substantial number of ginsenosides have been found to improve the decline in memory induced by lipopolysaccharide or okadaic acid (16-19). However, the effects of ginsenosides on memory decline induced by genetic interruption have not been reported, particularly its mechanisms. A previous study suggested that ginsenoside $\mathrm{Rg} 1$ is able to pass through blood-brain barrier to distribute in the cortex and hippocampus $(20,21)$. In the present study, 
an AD transgenic mouse model (APPswe/PSEN1dE9) was used to investigate the effects of ginsenoside Rg1 on memory, and to examine its underlying mechanisms. In combination with other evidence $(22,23)$, the present study hypothesized that ginsenoside $\mathrm{Rg} 1$ is a candidate memory enhancer, not only in age-related and drug-induced memory decline, but also in the genetic AD model. The present study may provide novel evidence to suggest a therapeutic effect of ginsenoside Rg1 on AD.

\section{Materials and methods}

Animals. APP/PS1 mice ( $\mathrm{n}=80$; B6C3-Tg) were obtained from the Jackson Laboratory (Farmington, CT, USA) and were bred amongst the colony. The offspring were genotyped using primers for APP and PS1 (Sangon Biotech Co., Ltd., Shanghai, China), which were as follows: Sense, 5'-GACTGACCACTC GACCAGGTTCTG-3' and antisense, 5'-CTTGTAAGTTGG ATTCTCATATCCG-3' for APP; sense, 5'-AATAGAGAA CGGCAGGAGCA-3' and antisense, 5'-GCCATGAGGGCA CTAATCAT-3' for PS1 reference; and sense, 5'-CCTCTT TGTGACTATGTGGACTGATGTCGG-3' and antisense, 5'-GTGGATAACCCCTCCCCCAGCCTAGACC-3' also for PS1, which distinguishes AD. C57 BL/6J mice $(n=30)$ were purchased from the Animal Center of the Chinese Academy of Sciences (Shanghai, China). The mice (male; age, 6 months; weight, $30 \mathrm{~g}$ ) used in the experiments were housed together in a $12 \mathrm{~h}$ light/dark cycle at $22 \pm 3^{\circ} \mathrm{C}$, with food and water ad libitum. All experimental procedures were approved by the ethics committee of Weifang Medical University (Weifang, China).

Ginsenoside Rgl treatment. APP/PS1 mice were chronically administered with Rg1 (Sigma-Aldrich, St. Louis, MO, USA) by intraperitoneal injection, at concentrations of 0.1 , 1 or $10 \mathrm{mg} / \mathrm{kg}$ once each day for 30 days consecutively. This concentration range was selected based on previous publications $(22,24)$. The control mice received the same volume of saline. During the drug administration, diet, water intake and body weights were monitored. At 30 days post-administration, behavioral, electrophysiological and biochemical experiments were performed.

Electrophysiological experiments. After 30 days, at least 4 mice from each group were sacrificed by decapitation. From each group, 4-8 slices were prepared. Acute hippocampal slices $(300 \mu \mathrm{m})$ were prepared following decapitation in cutting solution (Beyotime Institute of Biotechnology, Haimen, China). The components of the cutting solution were as follows: $124 \mathrm{mM} \mathrm{NaCl}, 26 \mathrm{mM} \mathrm{NaHCO}$, $10 \mathrm{mM}$ D-glucose, $3 \mathrm{mM}$ $\mathrm{KCl}, 1.25 \mathrm{mM} \mathrm{KH}_{2} \mathrm{PO}_{4}, 5 \mathrm{mM} \mathrm{MgSO}$ and $3.4 \mathrm{mM} \mathrm{CaCl}_{2}$. The slices were then transferred to an interface recording chamber (BSC-ZT; Warner Instruments LLC, Hamden, CT, USA) and exposed to a warm, humidified atmosphere of $95 \%$ $\mathrm{O}_{2} / 5 \% \mathrm{CO}_{2}$ and continuously perfused (for $\sim 4 \mathrm{~h}$ ) with oxygenated and preheated $\left(32 \pm 0.5^{\circ} \mathrm{C}\right)$ artificial cerebrospinal fluid (aCSF; Beyotime Institute of Biotechnology, Inc.) comprising $110 \mathrm{mM} \mathrm{NaCl}, 5 \mathrm{mM} \mathrm{KCl}, 2.5 \mathrm{mM} \mathrm{CaCl}_{2}, 1.5 \mathrm{mM} \mathrm{MgSO}_{4}$, $1.24 \mathrm{mM} \mathrm{KH}_{2} \mathrm{PO}_{4}, 10 \mathrm{mM}$ D-glucose and $27.4 \mathrm{mM} \mathrm{NaHCO}_{3}$. The aCSF flow speed was adjusted to $1.4 \mathrm{ml} / \mathrm{min}$. Following a $2 \mathrm{~h}$ recovery period, the field-excitatory postsynaptic potential (fEPSP), elicited by stimulation of the Schaffer collateral pathway with twisted nichrome wires (Warner Instruments LLC), was recorded. The input-output and paired-pulse facilitation at 30, 50 and $100 \mathrm{msec}$ intervals were assessed. Long-term potentiation was induced using a $\theta$-burst stimulation (TBS) protocol. Long-term depression (LTD) was induced by low-frequency stimulation (LFS).

ELISA. To quantify levels the of $\beta$-amyloid 1-42, the hippocampus from four sacrificed mice from each of the groups were homogenized in homogenization buffer ( $5 \mathrm{M}$ guanidine $\mathrm{HCl} / 50 \mathrm{mM}$ Tris-HCl; Beyotime Institute of Biotechnology) and centrifuged at $10,000 \mathrm{x}$ g for $10 \mathrm{~min}$ at $4^{\circ} \mathrm{C}$. The protein concentrations of the supernatants were determined using a Bicinchoninic Acid (BCA) Assay kit (Thermo Fisher Scientific, Inc., Waltham, MA, USA). The supernatant fractions were analyzed using a $\beta$-amyloid 1-42 ELISA kit (cat no. KHB3441; Invitrogen; Thermo Fisher Scientific, Inc.), according to the manufacturer's protocol. The absorbance was determined for each well at $450 \mathrm{~nm}$ using a microplate reader (Fluoroskan Ascent $^{\mathrm{TM}}$; Thermo Fisher Scientific, Inc.).

Reverse transcription-quantitative polymerase chain reaction (RT-qPCR) analysis. Total RNA was extracted from the hippocampus using TRIzol reagent (Invitrogen; Thermo Fisher Scientific, Inc.). Reverse transcription was performed using Moloney murine leukemia virus reverse transcriptase (Promega, Madison, WI, USA). RNA purity was defined by optical density (OD) ${ }_{260} / \mathrm{OD}_{280}$ on a Fluoroskan Ascent ${ }^{\mathrm{TM}}$ microplate reader. qPCR was performed to quantify the expression of APP in the hippocampus, using a quantitative thermal cycler (Mastercyclerep realplex; Eppendorf, Hamburg, Germany). The system included $2 \mu \mathrm{l}$ cDNA, $2 \mu 1$ dNTPs, $2 \mu 1 \mathrm{MgCl}_{2}$ and $\mathrm{ddH}_{2} \mathrm{O}$ to $25 \mu \mathrm{l}$. The thermocycling conditions were as follows: Initial denaturation, 5 min at $95^{\circ} \mathrm{C}$; and 30 cycles of denaturation at $30 \mathrm{sec}$ at $95^{\circ} \mathrm{C}$, annealing at $58^{\circ} \mathrm{C}$ for $30 \mathrm{sec}$ and extension at $72^{\circ} \mathrm{C}$ for $30 \mathrm{sec}$. The relative expression values were calculated as a ratio of target cDNA to $\beta$-actin and the expression of target genes was calculated by $2^{-\Delta \Delta \mathrm{Cq}}(25)$. The primers used in qPCR were obtained from Sangon Biotech Co., Ltd. as follows: APP, sense 5'-TGCTGG CAGAACCCCAGATCG-3' and antisense 5'-TTCTGGATG GTCACTGGCTGG-3'; $\beta$-actin sense 5-ATGAGGTAGTCT GTCAGGT-3 and antisense 5-ATGGATGACGATATCGCT-3.

Western blot analysis. The whole hippocampus homogenates were obtained and lysed, and the protein concentrations were measured using a BCA protein assay kit (Thermo Fisher Scientific, Inc.), as described above. Equivalent quantities of proteins $(20 \mu \mathrm{g})$ were processed for $12 \%$ SDS-PAGE (Beyotime Institute of Biotechnology) and western blot analysis. The proteins were transferred to nitrocellulose membranes (Bio-Rad Laboratories, Inc., Hercules, CA, USA) using a wet transfer and the membranes were blocked in 5\% nonfat milk for $2 \mathrm{~h}$ and washed three times in phosphate-buffered saline with Tween 20 (PBST). The membrane was incubated with primary antibodies overnight at $4^{\circ} \mathrm{C}$, as follows: Rabbit polyclonal BDNF (1:1,000; EMD Millipore, Billerica, MA, USA; cat. no. AB1534SP), rabbit actin (1:10,000; EMD Millipore; 
cat. no. MAB1501), rabbit monoclonal phosphorylated (p)-TrkB (1:3,000; Cell Signaling Technology, Inc., Danvers, MA, USA; cat. no. 4619), rabbit monoclonal Trk B (1:3,000; Cell Signaling Technology, Inc.; cat. no. 4607), rabbit polyclonal p-Tau (1:3,000; Cell Signaling Technology, Inc.; cat. no. 11834), rabbit monoclonal Tau (1:3,000; Cell Signaling Technology, Inc.; cat. no. 4019), C-terminal fragments (CTFs; 1:1,000; EMD Millipore; cat. no. AB5352), rabbit polyclonal postsynaptic density protein 95 (PSD-95; 1:3,000; Cell Signaling Technology, Inc.; cat. no. 2507) and rabbit polyclonal synaptophysin (1:3,000; Cell Signaling Technology, Inc.; cat. no. 4329). Following incubation with primary antibodies, the membranes were washed with PBST 3 times for $10 \mathrm{~min}$ and then incubated with the mouse anti-rabbit monoclonal secondary antibody (1:10,000; Cell Signaling Technology, Inc.; cat. no. 5127) for $2 \mathrm{~h}$ at room temperature. Protein levels were quantified by densitometry analysis using Quantity One software (version 4.5.2; Bio-Rad Laboratories, Inc.).

Fear conditioning. The fear conditioning experiment was performed, as previously described (26). The mice were handled daily for 5 days consecutively prior to training. On the training day, the mice were placed in the fear-conditioning chamber and allowed 5 min for exploration. Subsequently, three tone-footshock pairings, separated by 1 min intervals were delivered to the animals. The footshocks were $0.70 \mathrm{~mA}$ for $2 \mathrm{sec}$ and a tone of $85 \mathrm{~dB} 2 \mathrm{kHz}$ for $30 \mathrm{sec}$. The mice were retained in the training chamber for another $30 \mathrm{sec}$, following which they were transferred to their home cages. A context assessment $(5 \mathrm{~min})$ was performed $24 \mathrm{~h}$ post-training. On day 3 , the animals were subjected to a tone test in the same conditioning chamber, which was modified by a change in the color of the walls. The freezing level $(5 \mathrm{~min})$ in this altered context was measured (moving frequency, $<25 \mathrm{msec}$ ), and a tone $(85 \mathrm{~dB} ; 2 \mathrm{kHz})$ was delivered for $1 \mathrm{~min}$ to measure freezing to tone. The frequency of freezing was recorded using FreezeFrame software (version 3; Coulbourn Instruments, Holliston, MA, USA) and analyzed using FreezeView software (version 3; Coulbourn Instruments). In each group, there were five animals. The percentage of time in which the animal froze was calculated.

Statistical analysis. Data are presented as the mean \pm standard error of the mean. All statistical analyses were performed using one-way analysis of variance with GraphPad Prism 6.0 software (GraphPad Software, Inc., La Jolla, CA, USA). Bonferroni's correction with a post-hoc $t$-test was performed to compare the differences between groups. $\mathrm{P}<0.05$ was considered to indicate a statistically significant difference.

\section{Results}

Chronic treatment with ginsenoside Rgl ameliorates long-term memory in AD model mice. In the present study, long-term memory was measured using a fear conditioning experiment. Ginsenoside $\operatorname{Rg} 1$ was administered to the mice at a range of doses $(10,1$ and $0.1 \mathrm{mg} / \mathrm{kg}$ ) for 30 days. The dietary intake, drinking and body weights of the animals were unaffected during the drug treatment. As shown in Fig. 1A, context memory was markedly improved following treatment with
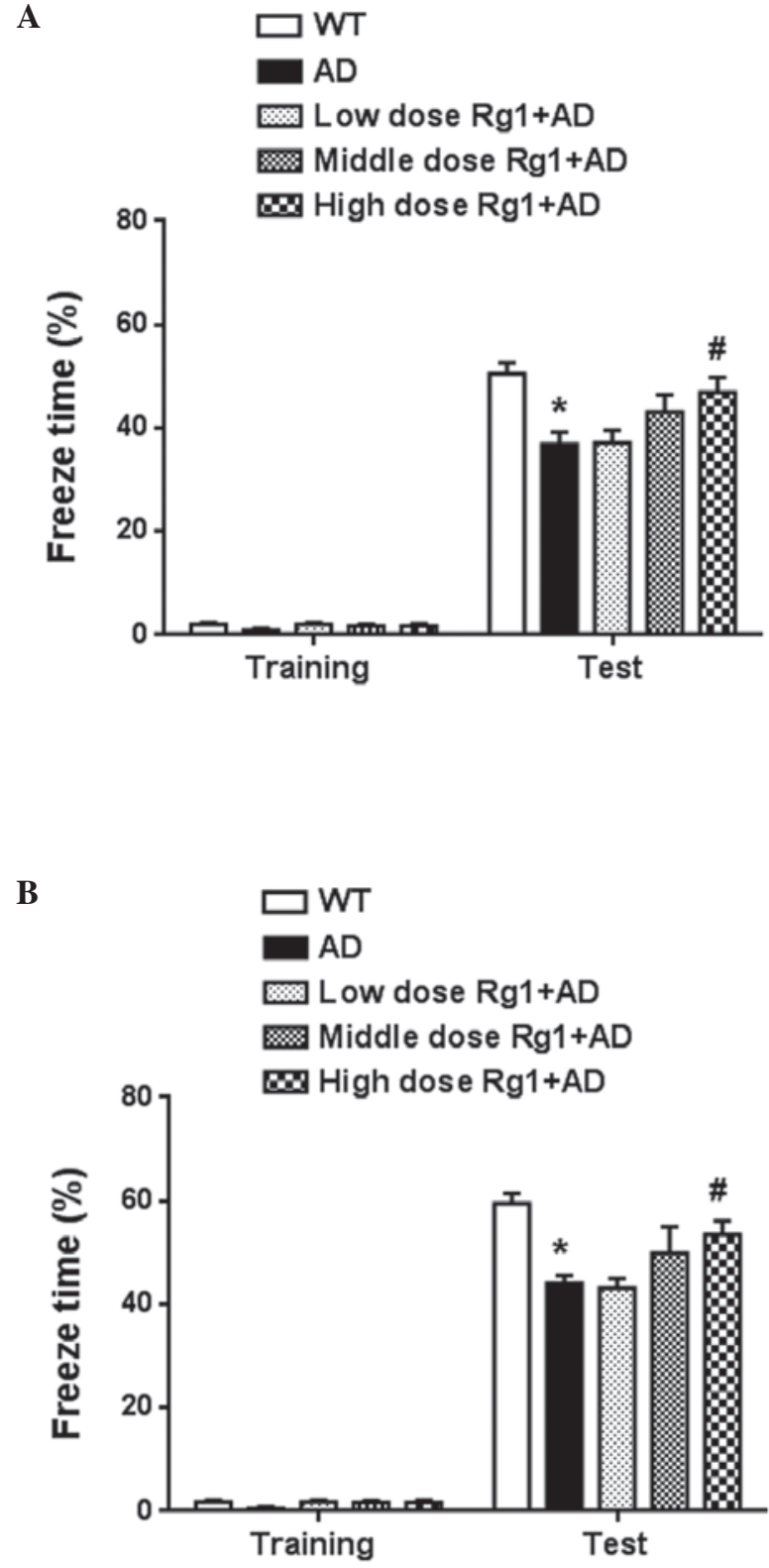

Figure 1. Chronic treatment with ginsenoside Rg1 improves memory in transgenic AD mice. (A) Context. (B) Tone. Each group comprised five animals. High $(10 \mathrm{mg} / \mathrm{kg})$, middle $(1 \mathrm{mg} / \mathrm{kg})$ and low $(0.1 \mathrm{mg} / \mathrm{kg})$ doses of ginsenoside $\mathrm{Rg} 1$ were administered to the mice for a consecutive 30 day period, respectively. Data are presented at the mean \pm standard error of the mean. ${ }^{*} \mathrm{P}<0.05$, compared with WT; ${ }^{\#} \mathrm{P}<0.05$, compared with AD. AD, Alzheimer's disease; WT, wild-type.

$10 \mathrm{mg} / \mathrm{kg}$ ginsenoside $\operatorname{Rg} 1(\mathrm{P}<0.05)$. The intermediate dose showed improved memory, but without statistical significance $(\mathrm{P}>0.05)$. No significant effect was observed following treatment with the low dose of ginsenoside Rg1. Tone memory was also measured. As shown in Fig. 1B, treatment with ginsenoside Rg1 at the dose of $10 \mathrm{mg} / \mathrm{kg}$ improved tone memory $(\mathrm{P}<0.05)$. The intermediate dose of ginsenoside $\mathrm{Rg} 1$ also had an ameliorating effect. These results confirmed that ginsenoside Rg1 improved long-term memory in the transgenic AD model.

Chronic treatment with ginsenoside Rg1 reverses LTP deficit in the AD model. To confirm the effect of ginsenoside $\mathrm{Rg} 1$ on hippocampal synaptic transmission and plasticity, the 
A

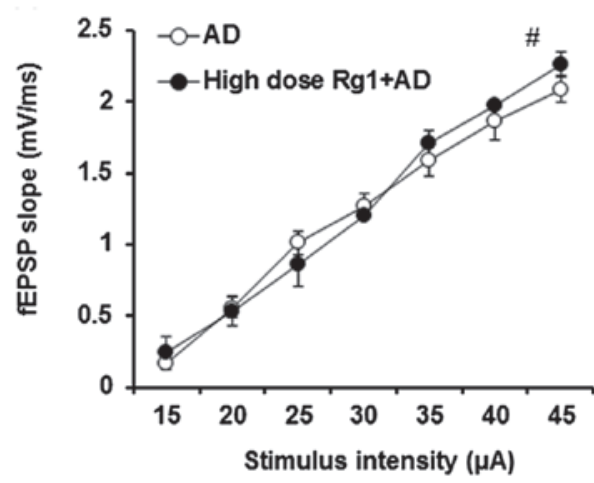

C

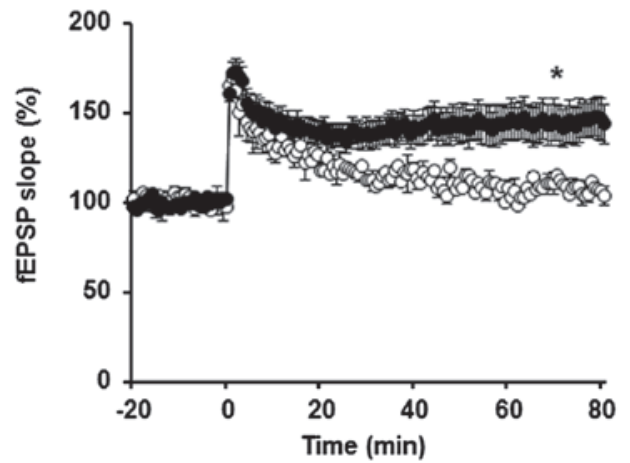

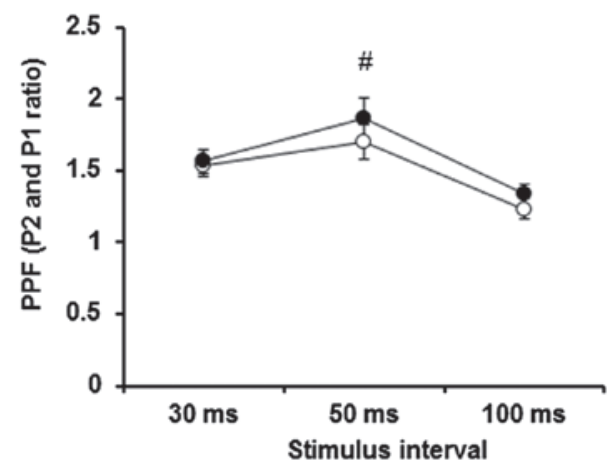

D

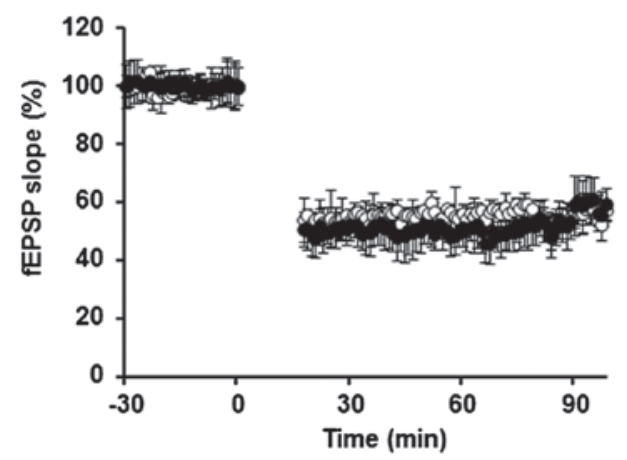

Figure 2. Chronic treatment with ginsenoside Rg1 increases LTP. (A) Input-output was not affected by ginsenoside Rg1 treatment. (B) PPF was not affected by ginsenoside Rg1 treatment. (C) TBS-induced LTP was improved by ginsenoside Rg1 treatment. (D) LFS-induced LTD was not affected by ginsenoside Rg1 treatment. Data are presented at the mean + standard error of the mean. In each group, 5-10 slices from five animals were included. ${ }^{*} \mathrm{P}<0.05$, ${ }^{\#} \mathrm{P}>0.05 \mathrm{vs}$. the AD group. AD, Alzheimer's disease; TBS, $\theta$-burst stimulation; LTP, long-term potentiation; LFS, low-frequency stimulation; LTD, long-term depression; fEPSP. field-excitatory postsynaptic potential; PPF, paired-pulse facilitation.

A

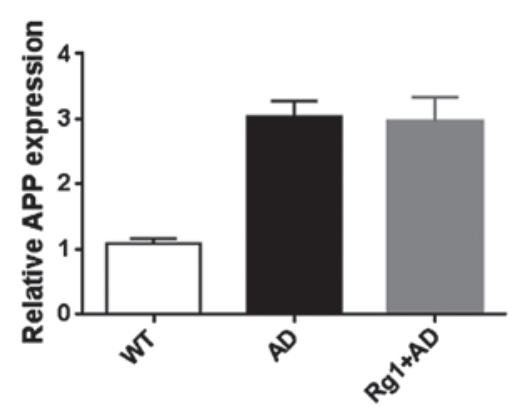

C

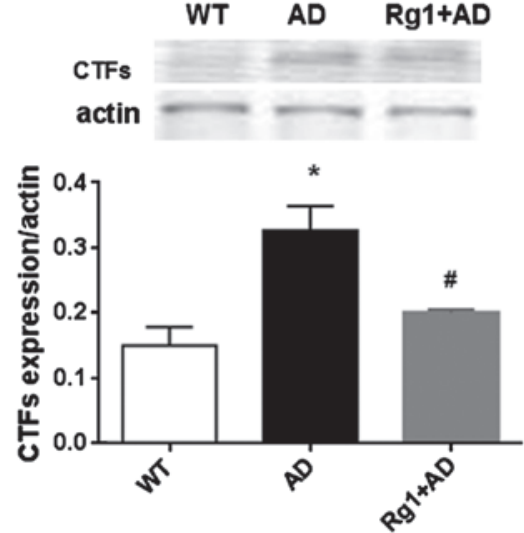

B

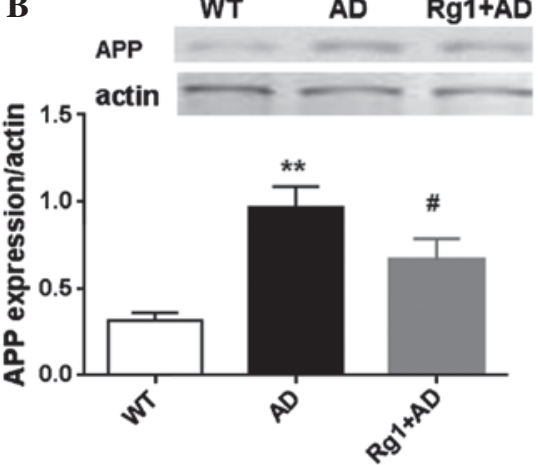

D
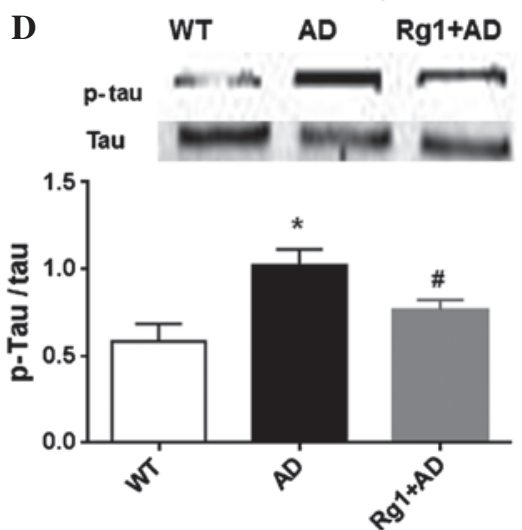

Figure 3. Chronic treatment with ginsenoside Rg1 downregulates the expression levels of APP, CTFs and p-Tau. (A) mRNA expression of APP; (B) protein expression of APP. Representative blots of APP and Actin are shown above. (C) Expression of CTFs. Representative blots of CTFs and Actin are shown above. (D) Expression of p-Tau. Representative blots of p-Tau and total Tau are shown above. Data are presented as the mean \pm standard error of the mean. Data were obtained from five animals in each group. " $\mathrm{P}<0.05$ and ${ }^{* *} \mathrm{P}<0.01$, compared with $\mathrm{WT} ;{ }^{~} \mathrm{P}<0.05$, compared with AD. APP, amyloid precursor protein; CTFs, C-terminal fragments; p-Tau, phosphorylated Tau; AD, Alzheimer's disease; WT, wild-type. 


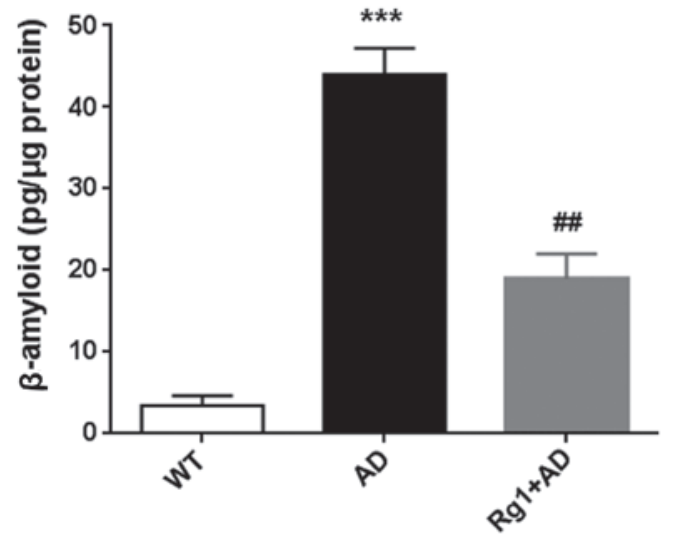

Figure 4. Chronic treatment with ginsenoside Rg1 downregulates levels of $\beta$-amyloid 1-42 in the AD model. Data are presented at the mean \pm standard error of the mean from five animals in each group. ${ }^{* * *} \mathrm{P}<0.001$, compared with $\mathrm{WT} ;{ }^{\# \#} \mathrm{P}<0.01$, compared with AD. AD, Alzheimer's disease; WT, wild-type.

fEPSPs at Schaffer collateral-CA1 synapses were measured. As shown in Fig. 2A and B, ginsenoside Rg1 had no effect on the input-output. Paired-pulse facilitation was also unaffected by ginsenoside $\mathrm{Rg} 1$ treatment $(\mathrm{P}>0.05)$. The induction of LTP by TBS was impaired in the slices obtained from the AD mice (Fig. 2C). However, following ginsenoside Rg1 treatment, TBS-LTP was ameliorated $(\mathrm{P}<0.05)$, compared with in the AD model. LFS-LTD was not affected by ginsenoside Rg1 treatment (Fig. 2D).

Chronic treatment with ginsenoside Rgl attenuates the expression of AD-associated proteins. The expression levels of APP and PS1 in the hippocampus were measured using RT-qPCR and Western blot analyses. Compared with the wild-type mice, the mRNA expression of APP increased $\sim 3$-fold in the AD model mice (Fig. 3A; P<0.05). Ginsenoside Rg1 did not alter the mRNA expression levels of APP. The protein levels were also determined. As shown in Fig. 3B, the protein level of APP also appeared to be enhanced in the AD model mice, compared with the wild-type mice. Of note, ginsenoside Rg1 decreased the protein levels following 1 month of treatment $(\mathrm{P}<0.05)$. In addition, the present study detected the expression of CTFs. In the model mice, the expression of CTFs was significantly increased $(\mathrm{P}<0.05)$, however, the expression was reduced by ginsenoside $\operatorname{Rg} 1$ treatment $(\mathrm{P}<0.05$; Fig. $3 \mathrm{C})$. The expression of $\mathrm{p}$-Tau was also measured. Compared with the wild-type mice, the expression of $\mathrm{p}$-Tau was increased in the model mice $(\mathrm{P}<0.05)$. Following treatment with ginsenoside $\mathrm{Rg} 1$, the protein level was also attenuated $(\mathrm{P}<0.05$; Fig. 3D). The level of $\beta$-amyloid 1-42 was reduced following treatment with ginsenoside Rg1 (Fig. 4). These results suggested that ginsenoside $\mathrm{Rg} 1$ treatment ameliorated the accumulation of $\mathrm{AD}$-associated proteins in the $\mathrm{AD}$ model mice.

Chronic treatment with ginsenoside RgI improves activation of the BDNF-TrkB pathway in AD model mice. Synaptic-associated proteins in the hippocampus were also measured in the present study, including BDNF, p-TrkB, synaptophysin and PSD-95. As shown in Fig. 5A and B, the expression of BDNF increased following treatment with ginsenoside $\operatorname{Rg} 1(\mathrm{P}<0.05)$. Correspondingly, the level of
A

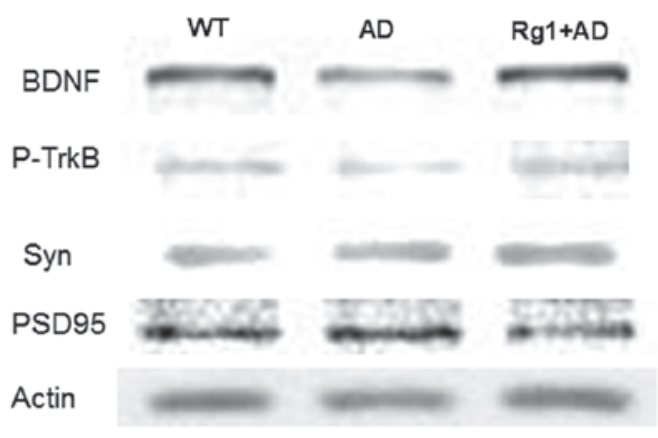

B

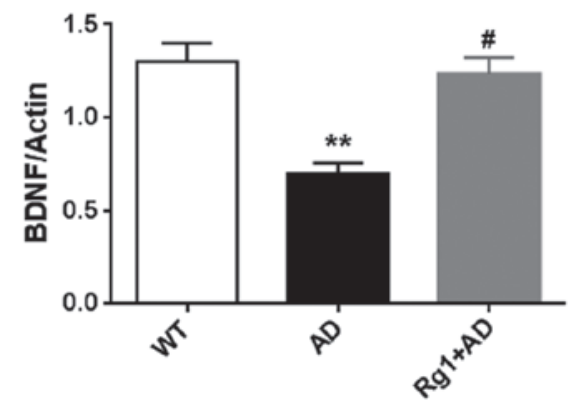

C

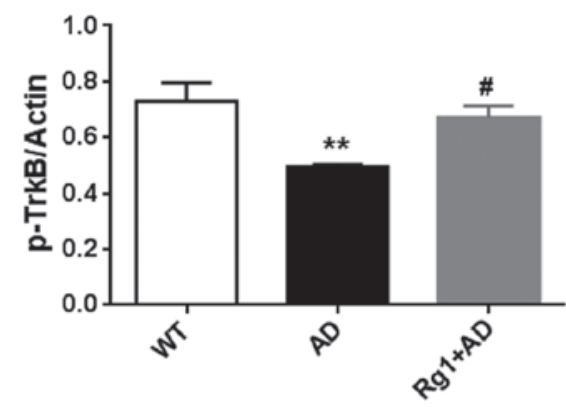

D

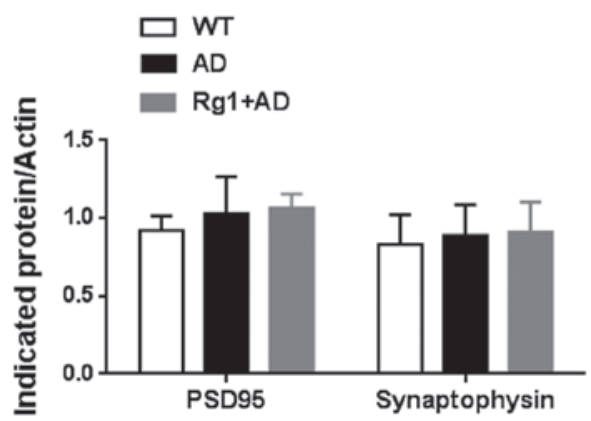

Figure 5. Chronic treatment with ginsenoside $\operatorname{Rg} 1$ activates the expression of BDNF and p-TrkB. (A) Representative blots of BDNF, p-TrkB, synaptophysin and PSD95. (B) Quantification of data of the expression of BDNF. (C) Quantification of data of the expression of p-TrkB. (D) Quantification data of the expres asion levels of synaptophysin and PSD95. Data are presented as the mean \pm standard error of the mean from five animals in each group. ${ }^{* *} \mathrm{P}<0.01$, compared with $\mathrm{WT}$; ${ }^{\#} \mathrm{P}<0.05$, compared with $\mathrm{AD}$. BDNF, brain-derived neurotrophic factor; $\mathrm{p}$-TrkB. phosphorylated tropomyosin receptor kinase B; Syn, synaptophysin; PSD95, postsynaptic density protein 95; AD, Alzheimer's disease; WT, wild-type.

$\mathrm{p}$-TrkB was also upregulated following treatment with ginsenoside Rg1 ( $\mathrm{P}<0.05$; Fig. 5C). By contrast, no effects were observed on the presynaptic marker, synaptophsin or post- 
synaptic marker, PSD-95 (Fig. 5D) following treatment with ginsenoside $\mathrm{Rg} 1$. These results indicated that ginsenoside $\mathrm{Rg} 1$ may have improved plasticity, but did not alter basal synapses in the AD model.

\section{Discussion}

In the present study, it was demonstrated that ginsenoside $\operatorname{Rg} 1$ treatment improved memory and hippocampal LTP in the $\mathrm{AD}$ model. The expression levels of $\mathrm{AD}$-associated proteins were attenuated, and the BDNF-TrkB pathway was improved following ginsenoside $\operatorname{Rg} 1$ treatment.

Ginsenoside Rgl ameliorates long-term memory in pathological disease models. A series of studies have reported that ginsenosides improve memory in exogenous toxin-induced memory deficits $(16-19,27,28)$. In addition, ginsenosides also improve memory in aging or aged animals $(23,29,30)$. These data indicate that ginsenosides are effective in improving memory. In the present study, ginsenoside $\operatorname{Rg} 1$ was selected as the target therapeutic drug, and a transgenic AD model was used to screen the effective doses. The results of the present and previous studies demonstrated that the APP/PS1 transgenic mice exhibited a decline in memory performance at 6 months of age (25). In the present study, intraperitoneal injection of $\operatorname{Rg} 1$ at concentrations between 1 and $10 \mathrm{mg} / \mathrm{kg}$ was selected, based on previous publications $(22,24)$. As shown by Zhang et al (22), this dose range is normal in mice and rats, following conversion from humans. Chronic treatment for 1 month with $10 \mathrm{mg} / \mathrm{kg}$ ginsenoside $\mathrm{Rg} 1$ significantly ameliorated long-term memory. Although the low dose of ginsenoside $\mathrm{Rg} 1(0.1 \mathrm{mg} / \mathrm{kg})$ did not cause amelioration in the AD model, the middle dose $(1 \mathrm{mg} / \mathrm{kg})$ demonstrated a protective effect. These results showed a dose-dependent effect of ginsenoside $\operatorname{Rg} 1$ on memory. Due to the chemical structure of ginsenoside Rg1, effective technology to improve its capacity to pass through the blood brain barrier is urgently required. Although $\beta$-amyloid peptide 1-42-induced functional loss is ameliorated by ginsenoside $\operatorname{Rg} 1$ application $(24,31)$, the present study demonstrated a similar effect of ginsenoside Rg1 using a transgenic $\mathrm{AD}$ model and fear conditioning experiment. The present study also aimed to clarify the potential mechanisms underlying the memory improvement observed following ginsenoside Rg1 treatment. As no commercial ginsenoside $\operatorname{Rg} 1$ injection is available, an effective dose range for oral application requires screening for clinical practice.

Ginsenoside Rgl facilitates the clearance of AD-associated proteins. Amyloid plaques are considered to be a detrimental toxin, contributing to the impairment of hippocampal synaptic plasticity and to hippocampal cell death (7-9). In the APP/PS1 transgenic mice, APP was overexpressed, and led to an increase in the accumulation of amyloid 1-42 in the hippocampus. In addition, the AD protein, $\mathrm{p}$-Tau, was enhanced at 6 months of age. These abnormalities caused by the overexpression of APP and PS1 may be responsible for the subsequent memory decline. In ginsenoside Rg1-treated mice, the expression levels of APP and PS1 were unaffected. However, the accumulation of p-Tau and amyloid 1-42 in the hippocampus were significantly reduced. The decreases in p-Tau and amyloid 1-42 may be caused by two factors. Protein synthesis may have been inhibited by ginsenoside $\operatorname{Rg} 1$ treatment. This possibility is supported by a previous study, which showed that ginsenoside $\mathrm{Rg} 1$ inhibits amyloid generation through regulation of the transcription or translation of BACE1 (32), or via inhibition of $\gamma$-secretase activity (33). The activity of the protein degradation system was enhanced following treatment with ginsenoside $\mathrm{Rg} 1$, leading to the degradation of $\beta$-amyloid, however, further clarification of this is required.

The majority of previous studies have focused on the amelioration of ginsenoside $\operatorname{Rg} 1$ in the later stage of $\mathrm{AD}$. Ginsenoside Rg1 may prevent against $\beta$-amyloid plague accumulation to inhibit apoptosis (34-36).

Ginsenoside Rgl ameliorates synaptic plasticity in the AD mice model. In addition to the clearance of $\mathrm{AD}$-associated proteins, ginsenoside $\mathrm{Rg} 1$ also facilitated the recovery of long-term potentiation. Initially, ginsenoside $\operatorname{Rg} 1$ treatment did not affect basal synaptic transmission, in terms of input-output and paired-pulse facilitation. These results suggested that ginsenoside $\operatorname{Rg} 1$ did not affect basal synaptic transmission, either presynaptically or postsynaptically. These physiological data were consistent with the unaltered expression levels of PSD-95 and synaptophysin. By contrast, plasticity was enhanced following ginsenoside $\mathrm{Rg} 1$ treatment in the AD model. The effects on LTP may be due to the clearance of AD-associated proteins. The present study found that the expression of BDNF was upregulated by ginsenoside $\mathrm{Rg} 1$ treatment and, correspondingly, $\mathrm{p}$-TrkB was activated following ginsenoside $\mathrm{Rg} 1$ treatment. Therefore, activation of the BDNF-TrkB pathway may contribute to the recovery of LTP in the transgenic AD model. In a senescence-accelerated mouse prone 8 model, the levels of BDNF are also improved following treatment with ginsenoside Rg1 (37). These findings indicate the general pharmacological activity of ginsenoside $\operatorname{Rg} 1$ in the $\mathrm{AD}$ model. In addition, other synaptic plasticity-associated proteins, including NR1 and NR2B, are reported to be upregulated in the AD model to increase memory (38). How ginsenoside Rg1 functions in the hippocampus remains to be fully elucidated and, although the present study did not distinguish the potential target, estrogen receptors have been implicated (39).

In the present study, data indicating memory amelioration following ginsenoside $\mathrm{Rg} 1$ treatment were obtained in a transgenic AD model. Clearance of $\mathrm{AD}$-associated proteins and activation of the BDNF-TrkB pathway may contribute to the effect of ginsenoside Rg1 on hippocampal LTP. These results suggested that ginsenoside $\mathrm{Rg} 1$ may be a potential memory enhancer in the transgenic AD model.

\section{References}

1. Qiu C, Kivipelto M and von Strauss E: Epidemiology of Alzheimer's disease: Occurrence, determinants and strategies toward intervention. Dialogues Clin Neurosci 11: 111-128, 2009.

2. Salloway S: Current and future treatments for Alzheimer's disease. CNS Spectr 14 (8 Suppl 7): 4-7; discussion 16-18, 2009.

3. Yiannopoulou KG and Papageorgiou SG: Current and future treatments for Alzheimer's disease. Ther Adv Neurol Disord 6: 19-33, 2013.

4. Aisen PS, Cummings J and Schneider LS: Symptomatic and nonamyloid/tau based pharmacologic treatment for Alzheimer disease. Cold Spring Harb Perspect Med 2: a006395, 2012. 
5. LaFerla FM, Green KN and Oddo S: Intracellular amyloid-beta in Alzheimer's disease. Nat Rev Neurosci 8: 499-509, 2007.

6. Li M, Chen L, Lee DH, Yu LC and Zhang Y: The role of intracellular amyloid beta in Alzheimer's disease. Prog Neurobiol 83: 131-139, 2007.

7. Carter J and Lippa CF: Beta-amyloid, neuronal death and Alzheimer's disease. Curr Mol Med 1: 733-737, 2001.

8. Oakley H, Cole SL, Logan S, Maus E, Shao P, Craft J, Guillozet-Bongaarts A, Ohno M, Disterhoft J, Van Eldik L, et al: Intraneuronal beta-amyloid aggregates, neurodegeneration and neuron loss in transgenic mice with five familial Alzheimer's disease mutations: Potential factors in amyloid plaque formation J Neurosci 26: 10129-10140, 2006.

9. Chapman PF, White GL, Jones MW, Cooper-Blacketer D, Marshall VJ, Irizarry M, Younkin L, Good MA, Bliss TV, Hyman BT, et al: Impaired synaptic plasticity and learning in aged amyloid precursor protein transgenic mice. Nat Neurosci 2: 271-276, 1999.

10. Hung IC, Chang SS, Chang PC, Lee CC and Chen CY: Memory enhancement by traditional Chinese medicine? J Biomol Struct Dyn 31: 1411-1439, 2013.

11. May BH, Lu C, Lu Y, Zhang AL and Xue CC: Chinese herbs for memory disorders: A review and systematic analysis of classical herbal literature. J Acupunct Meridian Stud 6: 2-11, 2013.

12. Yang L, Zhang J, Zheng K, Shen H and Chen X: Long-term ginsenoside Rg1 supplementation improves age-related cognitive decline by promoting synaptic plasticity associated protein expression in C57BL/6J mice. J Gerontol A Biol Sci Med Sci 69 : 282-294, 2014

13. Gillis CN: Panax ginseng pharmacology: A nitric oxide link? Biochem Pharmacol 54: 1-8, 1997.

14. Lee CH and Kim JH: A review on the medicinal potentials of ginseng and ginsenosides on cardiovascular diseases. J Ginseng Res 38: 161-166, 2014

15. Nah SY: Ginseng ginsenoside pharmacology in the nervous system: Involvement in the regulation of ion channels and receptors. Frontiers in physiology 5: 98, 2014.

16. Lee B, Sur B, Park J, Kim SH, Kwon S, Yeom M, Shim I, Lee H and Hahm DH: Ginsenoside rg3 alleviates lipopolysaccharide-induced learning and memory impairments by anti-inflammatory activity in rats. Biomol Ther (Seoul) 21: 381-390, 2013

17. Song XY, Hu JF, Chu SF, Zhang Z, Xu S, Yuan YH, Han N, Liu Y, Niu F, He X and Chen NH: Ginsenoside Rg1 attenuates okadaic acid induced spatial memory impairment by the GSK3beta/tau signaling pathway and the Abeta formation prevention in rats. Eur J Pharmacol 710: 29-38, 2013

18. Chu S, Gu J, Feng L, Liu J, Zhang M, Jia X, Liu M and Yao D: Ginsenoside Rg5 improves cognitive dysfunction and beta-amyloid deposition in STZ-induced memory impaired rats via attenuating neuroinflammatory responses. Int Immunopharmacol 19: 317-326, 2014

19. Wang Y, Kan H, Yin Y, Wu W, Hu W, Wang M, Li W and Li W: Protective effects of ginsenoside Rg1 on chronic restraint stress induced learning and memory impairments in male mice. Pharmacol Biochem Behav 120: 73-81, 2014

20. Nah JJ, Hahn JH, Chung S, Choi S, Kim YI and Nah SY: Effect of ginsenosides, active components of ginseng, on capsaicin-induced pain-related behavior. Neuropharmacology 39: 2180-2184, 2000.

21. Long W, Zhang SC, Wen L, Mu L, Yang F and Chen G: In vivo distribution and pharmacokinetics of multiple active components from Danshen and Sanqi and their combination via inner ear administration. J Ethnopharmacol 156: 199-208, 2014

22. Zhang X, Wang J, Xing Y, Gong L, Li H, Wu Z, Li Y, Wang J, Wang Y, Dong L and Li S: Effects of ginsenoside Rg1 or $17 \beta$-estradiol on a cognitively impaired, ovariectomized rat model of Alzheimer's disease. Neuroscience 220: 191-200, 2012.
23. Zhu G, Wang Y, Li J and Wang J: Chronic treatment with ginsenoside Rg1 promotes memory and hippocampal long-term potentiation in middle-aged mice. Neuroscience 292: 81-89, 2015.

24. Quan Q, Wang J, Li X and Wang Y: Ginsenoside Rg1 decreases A $\beta(1-42)$ level by upregulating PPARgamma and IDE expression in the hippocampus of a rat model of Alzheimer's disease. PloS One 8: e59155, 2013.

25. Hong X, Liu J, Zhu G, Zhuang Y, Suo H, Wang P, Huang D, $\mathrm{Xu}$ J, Huang Y, Yu M, et al: Parkin overexpression ameliorates hippocampal long-term potentiation and $\beta$-amyloid load in an Alzheimer's disease mouse model. Hum Mol Genet 23: 1056-1072, 2014.

26. Zhu G, Liu Y, Wang Y, Bi X and Baudry M: Different patterns of electrical activity lead to long-term potentiation by activating different intracellular pathways. J Neurosci 35: 621-633, 2015.

27. Liu J, Yan X, Li L, Zhu Y, Qin K, Zhou L, Sun D, Zhang X, Ye R and Zhao G: Ginsennoside rd attenuates cognitive dysfunction in a rat model of Alzheimer's disease. Neurochem Res 37: 2738-2747, 2012

28. Qiu J, Li W, Feng SH, Wang M and He ZY: Ginsenoside Rh2 promotes nonamyloidgenic cleavage of amyloid precursor protein via a cholesterol-dependent pathway. Genet Mol Res 13: 3586-3598, 2014

29. Zhao H,Li Q, Pei X,Zhang Z, Yang R, Wang J and Li Y: Long-term ginsenoside administration prevents memory impairment in aged C57BL/6J mice by up-regulating the synaptic plasticity-related proteins in hippocampus. Behav Brain Res 201: 311-317, 2009.

30. Zhao HF, Li Q and Li Y: Long-term ginsenoside administration prevents memory loss in aged female C57BL/6J mice by modulating the redox status and up-regulating the plasticity-related proteins in hippocampus. Neuroscience 183: 189-202, 2011.

31. Wang XY, Chen J and Zhang JT: Effect of ginsenoside Rg1 on learning and memory impairment induced by beta-amyloid peptide (25-35) and its mechanism of action. Yao Xue Xue Bao 36: 1-4, 2001 (In Chinese).

32. Chen LM, Lin ZY, Zhu YG, Lin N, Zhang J, Pan XD and Chen XC: Ginsenoside $\operatorname{Rg} 1$ attenuates $\beta$-amyloid generation via suppressing PPARgamma-regulated BACE1 activity in N2a-APP695 cells. Eur J Pharmacol 675: 15-21, 2012

33. Fang F, Chen X,Huang T,Lue LF,Luddy JS and YanSS: Multi-faced neuroprotective effects of Ginsenoside Rg1 in an Alzheimer mouse model. Biochim Biophys Acta 1822:286-292, 2012.

34. Wang YH and Du GH: Ginsenoside Rg1 inhibits beta-secretase activity in vitro and protects against Abeta-induced cytotoxicity in PC12 cells. J Asian Nat Prod Res 11: 604-612, 2009.

35. Shi C, Zheng DD, Fang L, Wu F, Kwong WH and Xu J: Ginsenoside Rg1 promotes nonamyloidgenic cleavage of APP via estrogen receptor signaling to MAPK/ERK and PI3K/Akt. Biochim Biophys Acta 1820: 453-460, 2012.

36. Li X, Zhang X, Yuan H and Quan Q: Experimental research on effect of gensenoside Rg1 on expressions of P-Tau and caspase-3 in brain slices from AD model rats. Zhongguo Zhong Yao Za Zhi 35: 369-372, 2010 (In Chinese).

37. Shi YQ, Huang TW, Chen LM, Pan XD, Zhang J, Zhu YG and Chen XC: Ginsenoside Rg1 attenuates amyloid-beta content, regulates PKA/CREB activity and improves cognitive performance in SAMP8 mice. J Alzheimer's Dis 19: 977-989, 2010.

38. Li X, Liu Y, Zhang X, Yuan H and Quan Q: Effect of ginsenoside $\mathrm{Rg} 1$ on expressions of phosphory protein tau and $\mathrm{N}$-methyl-D-aspartate receptor subunits NR1 and NR2B in rat brain slice model of Alzheimer's disease. Zhongguo Zhong Yao Za Zhi 35: 3339-3343, 2010 (In Chinese).

39. Shi C, Na N, Zhu X and Xu J: Estrogenic effect of ginsenoside $\mathrm{Rg} 1$ on APP processing in post-menopausal platelets. Platelets 24 : 51-62, 2013. 